07

\title{
Образование множественных сбоев в изделиях электроники под действием протонов и нейтронов
}

\author{
() Н.А. Иванов, ${ }^{1}$ О.В. Лобанов, ${ }^{1}$ В.В. Пашук, ${ }^{1}$ М.О. Прыгунов, ${ }^{2}$ К.Г. Сизова ${ }^{3}$ \\ ${ }^{1}$ Петербургский институт ядерной фризики им. Б.П. Константинова \\ Национального исследовательского центра Курчатовский институт, \\ 188300 Гатчина, Ленинградская обл., Россия \\ 2 ООО „Радиоавтоматика“, \\ 111033 Москва, Россия \\ ${ }^{3}$ ООО НПЦ „Гранат“, \\ 194021 Санкт-Петербург, Россия \\ e-mail: ksizova@npcgranat.ru
}

Поступило в Редакцию 4 сентября 2019 г.

В окончательной редакции 4 сентября 2019 г.

Принято к публикации 14 октября 2019 г.

\begin{abstract}
Проведено облучение нуклонами на пучках синхроциклотрона Петербургского института ядерной физики (ПИЯФ) интегральных схем памяти типа SRAM с технологической нормой $90 \mathrm{~nm}$ и оптоэлектронных изделий: CCD- и CMOS-матриц. Представлены данные по сечениям образования сбоев в интегральных схемах памяти под действием протонов с энергией $1000 \mathrm{MeV}$ и пикселей с большой величиной темнового тока (спайков) в оптоэлектронных изделиях, облученных протонами с энергией $1000 \mathrm{MeV}$, и нейтронами с энергетическим спектром, подобным спектру атмосферных нейтронов. Основное внимание уделено исследованию возникновения кластеров спайков и множественных сбоев. Установлено, что основная часть спайков и одиночных сбоев находится в составе кластеров.
\end{abstract}

Ключевые слова: спайки, кластеры, множественные сбои, протоны, нейтроны, интегральные схемы, память, оптоэлектронные изделия.

DOI: 10.21883/JTF.2020.04.49095.311-19

\section{Введение}

Широкое применение интегральных схем (ИС) в изделиях космической и авиационной электронной техники сделало актуальной задачу обеспечения их надежного функционирования в условиях воздействия радиационных полей. В ИС, изготовляемых по микронной и субмикронной технологиям, наиболее существенным фактором, определяющим их нормальную работу при облучении быстрыми нуклонами и ионами, являются одиночные эффекты сбоя и отказа (SEE - Single-Event Effects) [1-3]. Данный эффект связан с однократным выделением одной частицей больших величин энергии в объеме чувствительных областей ИС, вследствие чего происходит потеря работоспособности отдельного элемента изделия (сбой, катастрофический отказ). В зависимости от типа ИС вероятность SEE-эффекта под действием частиц пропорциональна их линейным потерям энергии на ионизацию (ЛПЭ) [4] или на упругие столкновения с атомами полупроводникового материала - неионизационные энергетические потери (НИЭП) [5].

Первая работа, в которой был обнаружен эффект воздействия отдельных нуклонов, была опубликована в 1965 г. [6]. Начиная с 1980-х годов эффект воздействия отдельных частиц, как основной фактор, определяющий надежность работы электроники в космосе и ближней атмосфере, становится приоритетным направлением исследований воздействия излучения на различные типы ИС $[7,8]$. Продолжающееся снижение технологических норм изготовления ИС сопровождается [9]:

- уменьшением критических величин энергии, необходимых для возникновения сбоя (отказа) элемента изделия, что приводит к увеличению вероятности повреждения ИС под действием отдельных частиц, имеющих меньшее энерговыделение в чувствительной области;

- ростом вероятности повреждения нескольких соседних элементов ИС - возникновением множественных сбоев (МС) под действием отдельной частицы.

Настоящая работа направлена на исследование образования МС под действием быстрых нуклонов в оптоэлектронных изделиях и элементах памяти.

\section{1. Исследуемые интегральные схемы}

Использовались светочувствительные ИС:

- приборы с зарядовой связью (CCD-матрицы) ICX 259AL фирмы Sony с размером чипа $6 \times 4.96 \mathrm{~mm}$, размером пикселя $6.50 \times 6.25 \mu \mathrm{m}$ с числом активных элементов $\sim 4 \cdot 10^{5}$;

- матрицы на основе комплементарной структуры металл-оксид-полупроводник (CMOS-матрицы) MT9M034 фирмы Aptina ${ }^{\text {TM }}$ Imaging с размером чипа 
$4.8 \times 3.6 \mathrm{~mm}$, размером пикселя $3.75 \times 3.75 \mu \mathrm{m} \mathrm{c} \mathrm{чис-}$ лом активных элементов $10^{6}$.

Известно $[2,8,10]$, что в оптоэлектронных изделиях эффект воздействия отдельных частиц проявляется в возникновении спайков - пикселей с темновыми токами $I_{d c}$, существенно превышающими средние значения темновых токов в пикселях матриц. Такие пиксели на изображении с матрицы проявляются в виде белых точек, которые сохраняются и после окончания облучения. Создание спайков обусловлено образованием радиационных дефектов в чувствительной области пикселей и соответственно эффективность возникновения спайков пропорциональна величине НИЭП частиц.

Применение матриц для исследования МС сбоев обусловлено тем, что они имеют простую топологию расположения пикселей, что позволяет легко получать информацию о количестве и пространственном распределении поврежденных пикселей. Данная информация полезна для анализа экспериментальных данных о радиационном воздействии излучения на другие типы ИС.

В качестве ИС с низкими технологическими нормами использовалась статическая память типа SRAM производителя Cypress c технологической нормой $90 \mathrm{~nm}$. Данная ИС имеет площадь чипа $5.34 \mathrm{~mm}^{2}$, содержит свыше $4 \cdot 10^{6}$ bit. В отличие от повреждения матриц основной причиной возникновения сбоев в памяти SRAM являются ионизационные процессы в чувствительных областях ячеек. Соответственно эффективность образования сбоев зависит от величины ЛПЭ ионизирующих излучений.

\section{2. Экспериментальные методики}

Облучение матриц и элементов памяти SRAM проводилось на синхроциклотроне ПИЯФ [11] при энергии протонов $1000 \mathrm{MeV}$ и плотности потока $\sim 10^{8} \mathrm{~cm}^{-2} \cdot \mathrm{s}^{-1}$. Диаметр пучка протонов составлял $28 \mathrm{~mm}$ при неоднородности плотности потока до 5\%. Образцы ИС облучались на специализированном стенде, включающем:

- магнитную систему вывода пучка и лазерную систему фиксирования направления пучка;

- устройства мониторирования пучка протонов: автоматизированный измерительный комплекс на основе двухсекционной ионизационной камеры [12] для измерения плотности потока и флюенса протонов с погрешностью на уровне $5 \%$ и полупроводниковый $\mathrm{Ge}(\mathrm{Li})$ детектор для измерения потока протонов методом наведенной активности [13] с погрешностью до $30 \%$.

В соответствии с рекомендациями Международного нормативного документа „JEDEC STANDART“ [14] для облучения изделий электроники нейтронами должен использоваться пучок нейтронов с энергетическим спектром, подобным атмосферному спектру нейтронов. Нейтроны с таким энергетическим спектром с максимальной энергией $1000 \mathrm{MeV}$ были получены в ПИЯФ на установке ГНЕЙС (Гатчинский нейтронный спектрометр) [15] в 2010г. Облучение матриц на этой установке проводилось при плотности потока нейтронов $\sim 1.5 \cdot 10^{5} \mathrm{~cm}^{-2} \cdot \mathrm{s}^{-1}$, неоднородности в области расположения ИС менее $5 \%$ и погрешности измерения потока нейтронов 5\% [15].

Матрицы в составе телевизионных камер в процессе облучения нуклонами были в активном (с включенным питанием) или в пассивном состояниях (с выключенным питанием), с включенной или выключенной системой автоматической регулировки усиления (АРУ). Темновые токи $I_{d c}$ в пикселях определялись по степени яркости пикселя при анализе изображений на экране монитора компьютера с помощью программ Mathcad. Степень яркости пикселя матрицы оценивалась в относительных единицах (r.u.) целым числом в диапазоне от 0 (черное) до 255 (белое).

Два образца памяти SRAM были облучены протонами до флюенса $10^{11} \mathrm{~cm}^{-2}$. Методика исследования ИС заключалась в следующем:

- до начала облучения производилась запись шаблона данных в массив ячеек памяти;

- в процессе облучения контролировалось состояние каждого байта памяти;

- в процессе контроля над каждым байтом выполнялось три операции:

- первые две заключались в считывании данных и сравнении их с шаблоном;

- если только в одном из двух первых чтений фиксировалась ошибка, то в качестве третьей операции выбиралась также операция „чтение“ и ошибка классифицировалась как сбой в процессе чтения;

- если в двух первых чтениях фиксировалась ошибка, то ошибка классифицировалась как сбой, произошедший во время хранения, и производилась перезапись байта.

Обеспечение выполнения одинакового количества операций, производимым над каждым байтом, позволило равномерно распределить время хранения данных в каждом байте. За счет выборочной перезаписи поврежденных ячеек была достигнута минимизация количества выполнений операций записи в процессе воздействия, что позволило уменьшить период обработки байта и выдачи информации опрашивающего устройства до $2 \mathrm{~ms}$. Вследствие этого удалось уменьшить число сбоев в процессе перезаписи и соответственно снизить их влияние на экспериментальные данные.

\section{3. Экспериментальные результаты}

\section{1. Облучение CCD-матриц}

При облучении нейтронами и протонами образование спайков в CCD-матрицах происходит в основном под действием продуктов ядерных реакций нуклонов с ядрами атомов кремния $[2,8,10,16,17]$. В процессе облучения протонами телевизионной системы с CCD-матрицей в 

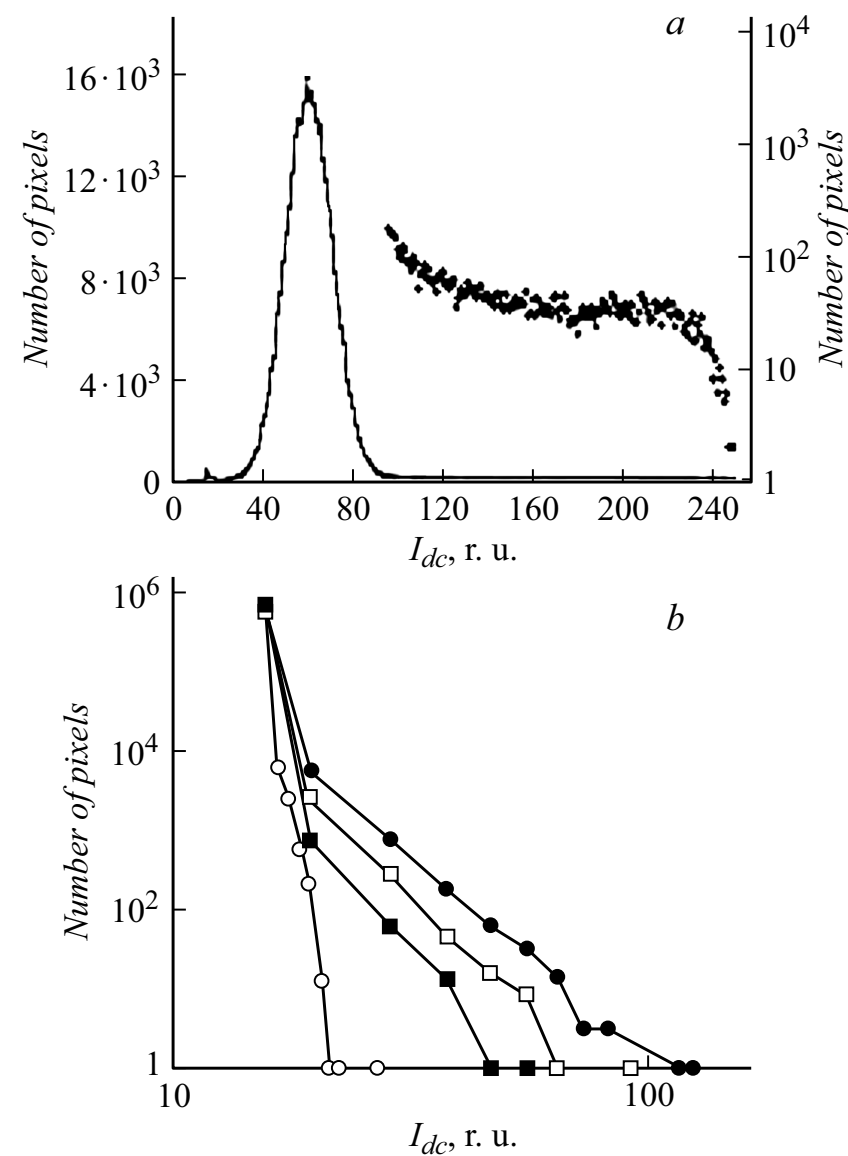

Рис. 1. Распределение по величине темнового тока в пикселях CCD-матрицы, облученной $a-$ протонами (APУ включено): - - исходная, $\bullet \bullet \bullet-$ флюенс $10^{10} \mathrm{~cm}^{-2}$, на правой шкале ординат показаны значения больших темновых токов в спайках; $b-$ нейтронами (АРУ выключено): о - исходная, флюенсы - $-5.3 \cdot 10^{8} \mathrm{~cm}^{-2}, \diamond-1.6 \cdot 10^{9} \mathrm{~cm}^{-2}, \bullet-$ $3.2 \cdot 10^{9} \mathrm{~cm}^{-2}$.

активном режиме на экране монитора компьютера появлялись кратковременные вспышки, обусловленные ионизационными потерями протонов в объеме пикселей [16]. Следует отметить, что были видны также следы (треки) заряженных частиц, образующихся в неупругих ядерных взаимодействиях протонов с ядрами материалов CCDматриц. Накапливающиеся по мере возрастания флюенса протонов устойчивые дефектные пиксели (спайки) в виде белых пятен оставались и после окончания облучения.

На рис. 1 приведены распределения пикселей по величине темнового тока в исходной и облученной CCDматрице в следующих условиях:

— флюенс протонов $10^{10} \mathrm{~cm}^{-2}$, система АРУ включена;

- флюенс нейтронов $3.2 \cdot 10^{9} \mathrm{~cm}^{-2}$, система АРУ выключена.

Из рисунка видно, что в необлученных матрицах при включенной АРУ распределение пикселей по величине $I_{d c}$ описывалось нормальным распределением со сред- ним значением $\approx 60$ r.u. и стандартным отклонением $\sigma \approx 10$ r.u., при этом не более 10 пикселей в матрице имели яркость свыше 110 r.u., что превышает максимум нормального распределения на $5 \sigma$. В режиме выключенной АРУ распределение пикселей по величине $I_{d c}$ имело вид кривой, резко спадающей с ростом темнового тока, и пиксели с величиной $I_{d c}$ более 20 r.u. практически отсутствовали. В связи с этим спайками мы считали пиксели, величина $I_{d c}$ в которых после облучения превышала в зависимости от состояния АРУ 110 или 20 r.u. Следует отметить, что в результате измерений не обнаружено существенных различий в параметрах матриц, облученных в активном и в пассивном режимах.

Для анализа пространственного распределения пикселей с большими величинами $I_{d c}$ (спайков) была создана программа для поиска областей CCD-матрицы, в пределах которых темновой ток каждого из пикселей превышал заданный порог. В качестве примера на рис. 2 представлено распределение таких областей по числу входящих в них пикселей с $I_{d c}>170$ r.u. (АРУ включено) после облучения нейтронами флюенсом $3.2 \cdot 10^{9} \mathrm{~cm}^{-2}$. Отбирались области, в которых не менее двух пикселей удовлетворяли заданному критерию. Наиболее частыми явились области из двух пикселей. Обращает на себя внимание наличие групп - кластеров спайков, включающих до 14 пикселей. В соответствии с результатами работ $[17,18]$ при облучении протонами и нейтронами основная часть спайков (свыше 50\%) входит в состав таких кластеров.

По всей видимости, возникновение данных кластеров обусловлено в основном тем, что в пикселях, удаленных на десятки микрон от точки ядерного взаимодействия, радиационные дефекты создаются остаточными ядрами из ядерных реакций. В табл. 1 приведены величины пробегов [4] ядер отдачи, образующихся с максимальными энергиями в ядерных реакциях протонов с энергией $1000 \mathrm{MeV}$ с ядрами атомов кремния [19]. Из этой таблицы видно, что такие ядра отдачи как неон, кислород

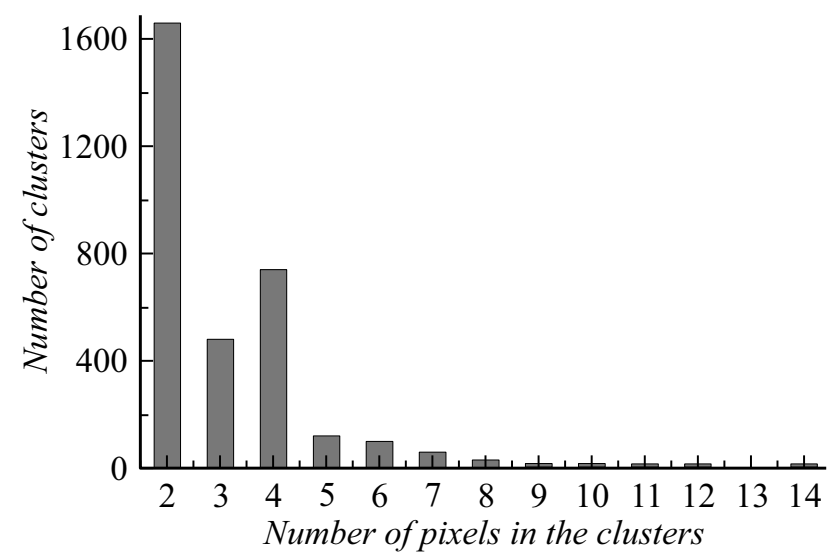

Рис. 2. Распределение по числу соседних пикселей, величина $I_{d c}$ в каждом из которых больше 170 r.u. (АРУ включено) в CCD-матрице, облученной нейтронами флюенсом $3.2 \cdot 10^{9} \mathrm{~cm}^{-2}$. 

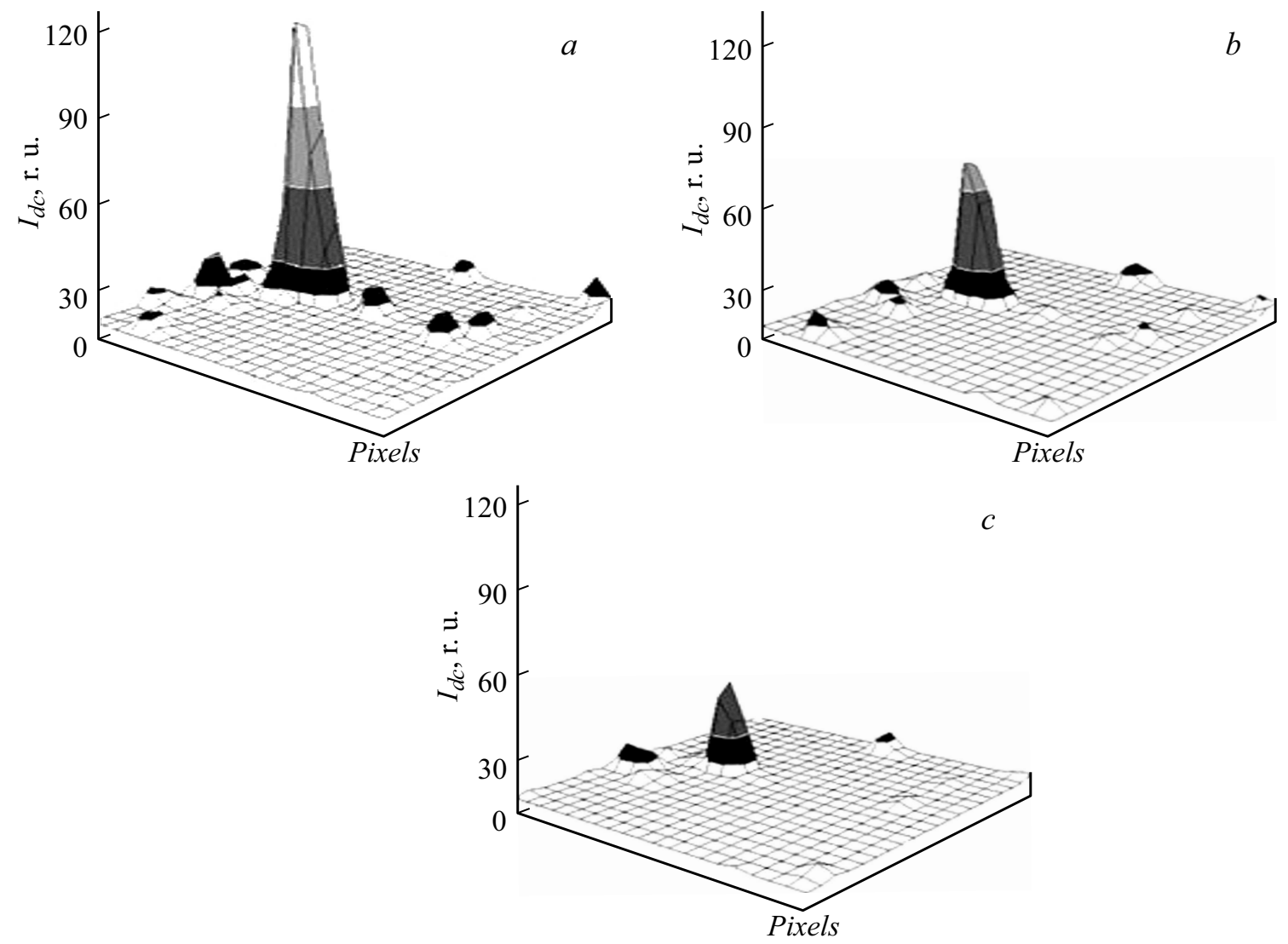

Рис. 3. Рельеф кластера спайков в CCD-матрице, облученной нейтронами с флюенсом $3.2 \cdot 10^{9} \mathrm{~cm}^{2}: a-$ после облучения: $b-$ через $32 \mathrm{~h}, c$ - через $214 \mathrm{~h}$.

Таблица 1. обеги ядер отдачи, образующихся с максимальными энергиями в ядерных реакциях нуклонов с энергией $1000 \mathrm{MeV}$ с ядрами атомов кремния $[4,19]$

\begin{tabular}{l|c|c|c|c}
\hline Тип ядра отдачи & ${ }^{27} \mathrm{Al}$ & ${ }^{20} \mathrm{Ne}$ & ${ }^{16} \mathrm{O}$ & ${ }^{12} \mathrm{C}$ \\
\hline Энергия ядра отдачи, $\mathrm{MeV}$ & 7.5 & 24 & 46 & 53 \\
\hline Пробег ядра, $\mu \mathrm{m}$ & 4.5 & 11 & 30 & 63
\end{tabular}

Таблица 2. Значения параметра $t_{0}$ при различной пороговой величине темнового тока

\begin{tabular}{c|c|c|c|c|c|c}
\hline$>I_{d c}$, r.u. & 100 & 125 & 150 & 175 & 200 & 225 \\
\hline$t_{0}, \mathrm{~s}$ & 179980 & 152295 & 136636 & 118083 & 100740 & 82085
\end{tabular}

и углерод имеют пробеги, достаточные для создания кластеров спайков наблюдаемых размеров. Следует также иметь в виду, что эти ядра отдачи в процессе торможения проходят область максимальных значений линейных потерь энергии на ионизацию и упругие атоматомные соударения [4,5].

После облучения протонами и нейтронами систематические измерения $I_{d c}$ проводились в течение нескольких месяцев. В промежутках между измерениями камера находилась в пассивном режиме при комнатной температуре и включалась непосредственно перед съемом информации. Вначале проводились измерения в режиме с включенной АРУ, затем - без АРУ. Полученные экспериментальные данные по количеству спайков $N$ в зависимости от времени $t$ после облучения были аппроксимированы экспоненциальной функцией: $N \sim \exp \left(-t / t_{0}\right)$. Величины параметра $t_{0}$ для различных пороговых значений $I_{d c}$ при измерениях в режиме с включенной системой АРУ приведены в табл. 2, из которой видно, что в среднем величина $t_{0}$ составляет около $10^{5} \mathrm{~s}$. Величина параметра $t_{0}$ при измерениях в режиме с выключенной системой АРУ составила около $4 \cdot 10^{4} \mathrm{~s}$. Полученные величины $t_{0}$ качественно согласуются с данными работ [20-22] для разных типов ПЗС-матриц.

Уменьшение количества спайков приводит к изменениям кластеров. В качестве примера на рис. 3 приведены рельефы одного и того же кластера, который непосредственно после облучения нейтронами с флюенсом $3.2 \cdot 10^{9} \mathrm{~cm}^{2}$ включал 8 спайков (рис. $\left.3, a\right)$. На рис. $3, b$, с показаны рельефы этого кластера при временах до 10 суток после окончания облучения. Из этих рисунков видно, что после облучения происходит уменьшение количества спайков в кластерах и поперечных размеров кластеров, а также снижается яркость спайков в 
кластере. Наблюдалось исчезновение со временем части кластеров, яркость спайков в которых уменьшалась до величин $I_{d c}$ в необлученной CCD-матрице.

Снижение количества спайков и их яркости обусловлено отжигом радиационных дефектов в облученных изделиях. Вследствие этого при длительном облучении нуклонами $\left(\gg t_{0}\right)$ в CCD-матрице должно создаваться равновесное количество спайков и кластеров, которое определяется из условия равенства скорости образования новых спайков и кластеров скорости их отжига. Полученные результаты указывают на влияние плотности потока частиц на распределение темнового тока в пикселях CCD-матриц. Этот фактор должен учитываться при прогнозировании эффектов от воздействия отдельных частиц по данным лабораторных испытаний на моделирующих установках (ускорителях), на которых плотность потока частиц на 3-4 порядка превышает плотности потоков частиц космического излучения.

\section{2. Облучение CMOS-матриц}

Интерес к исследованию CMOS-матриц обусловлен тем, что благодаря своим техническим характеристикам и низкой стоимости они в последнее десятилетие активно заменяют CCD-матрицы в электронной аппаратуре. CMOS-матрицы были облучены протонами до флюенса $10^{10} \mathrm{~cm}^{-2}$ и нейтронами до флюенса $2.8 \cdot 10^{7} \mathrm{~cm}^{-2}$. Величины $I_{d c}$ определялись, так же как и для CCD-матриц. Поскольку в исходных CMOS-матрицах величины $I_{d c}$ при измерениях без АРУ не превышали 20 r.u., спайками мы считали пиксели, величина $I_{d c}$ в которых после облучения превышала 20 r.u.

На рис. 4 приведены распределения количества кластеров по числу входящих в них спайков в облученных матрицах. В качестве примера на рис. 5 приведен участок матрицы с типичными „рельефами“ кластеров спайков после облучения CMOS-матрицы протонами и

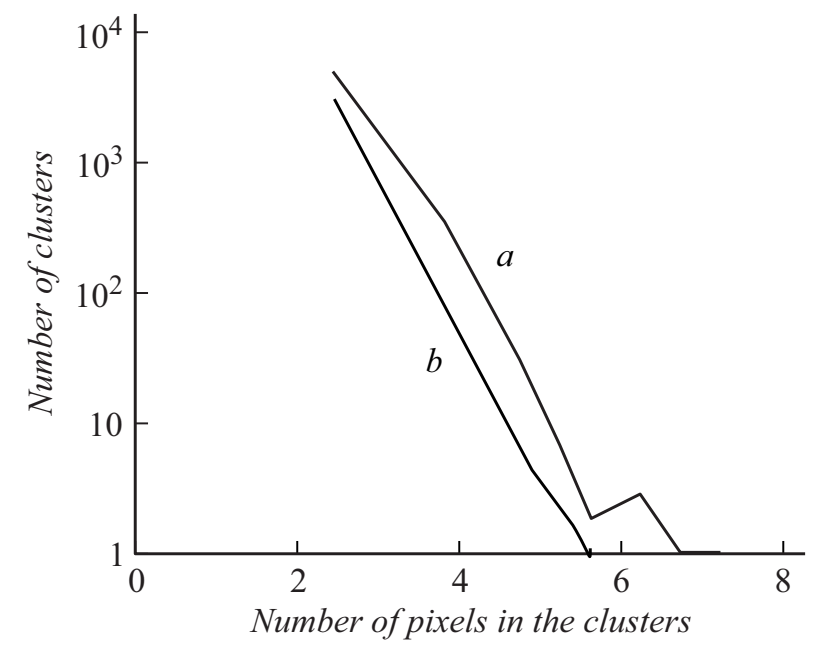

Рис. 4. Распределения количества кластеров по числу входящих в них спайков в матрицах, облученных протонами $(a)$ и нейтронами $(b)$. нейтронами. Видно, что размеры кластеров и величины темновых токов в спайках, входящих в их состав, практически не зависят от природы нуклонов. Максимальный размер кластеров, приведенных на рис. 5, составляет $\sim 11.75 \times 11.75 \mu \mathrm{m}$ и включает в себя 9 спайков. К образованию кластеров таких размеров, так же как и в CCD-матрицах, способны приводить длиннопробежные остаточные ядра из ядерных реакций нуклонов с ядрами кремния (табл. 1).

При флюенсе протонов $10^{10} \mathrm{~cm}^{-2}$ количество событий $n$ от отдельных частиц составило 28926 , в том числе 20194 кластеров и 8732 спайков вне кластеров. Соответственно сечение возникновения событий от отдельных протонов в CMOS-матрице $\Sigma_{\mathrm{sp}}$, равное $\Sigma_{\mathrm{sp}}=n / F_{p}$, где $F_{p}$ - флюенс протонов, составило $2.9 \cdot 10^{-6} \mathrm{~cm}^{-2}$, которое качественно согласуется с величиной сечения ядерных реакций в объеме CMOS-матрицы $\Sigma_{\mathrm{nr}}$ :

$$
\Sigma_{\mathrm{nr}}=\sigma n_{\mathrm{Si}} V_{\mathrm{CMOS}} \approx 2.2 \cdot 10^{-6} \mathrm{~cm}^{2}
$$

где $\sigma=5 \cdot 10^{-25} \mathrm{~cm}^{2}-$ сечение ядерных реакций нуклонов с энергией $1000 \mathrm{MeV}$ с ядрами атомов кремния [21]; $n_{\mathrm{Si}}=5.19 \cdot 10^{22} \mathrm{~cm}^{-3}$ - плотность атомов кремния; $V_{\mathrm{CMOS}}$ - объем CMOS-матрицы, принятый нами равным $0.86 \cdot 10^{-4} \mathrm{~cm}^{3}$ при толщине чувствительной области матрицы $5 \mu \mathrm{m}$.

Полученное соотношение - 20194 кластеров и 8732 спайков вне кластеров - указывает на то, что большая часть спайков (около 80\%) возникает в составе кластеров.

В разд. 3.1 и 3.2 было показано, что в CCD- и CMOSматрицах, облученных протонами и нейтронами, под действием продуктов ядерных реакций образуются локальные скопления (кластеры) с линейными размерами до $20 \mu \mathrm{m}$, состоящие из соседних поврежденных пикселей (спайков). Эти кластеры спайков являются, фактически, аналогом МС, что указывает на возможность возникновения МС под действием нуклонов в других типах ИС, имеющих близкие или меньшие значения размеров элементарной ячейки и/или чувствительной области, чем размеры пикселя матрицы.

\section{3. Облучение SRAM}

Два образца SRAM памяти были облучены протонами с энергией $1000 \mathrm{MeV}$ при плотности потока $10^{8} \mathrm{~cm}^{-2} \cdot \mathrm{s}^{-1}$ до флюенса $10^{11} \mathrm{~cm}^{-2}$. В образцах были обнаружены сбои, характеризуемые как ошибки хранения, а ошибок чтения не было зафиксировано. В результате исследования за весь сеанс облучения в образцах было обнаружено 16169 и 17414 ошибок хранения в различных байтах. Сечение сбоев $\Sigma$, равное $\Sigma=n /\left(F N_{\mathrm{bit}} C\right)$, где $n-$ количество сбоев, $F=10^{11} \mathrm{~cm}^{-2}$ - флюенс протонов, $N_{\mathrm{bit}}=4 \cdot 10^{6}-$ количество бит в ИС, составило $\sim 4 \cdot 10^{-14} \mathrm{~cm}^{-2} \cdot \mathrm{bit}^{-1}$, что согласуется с данными работы [23] для аналогичных по типу и технологическим нормам изделий. 

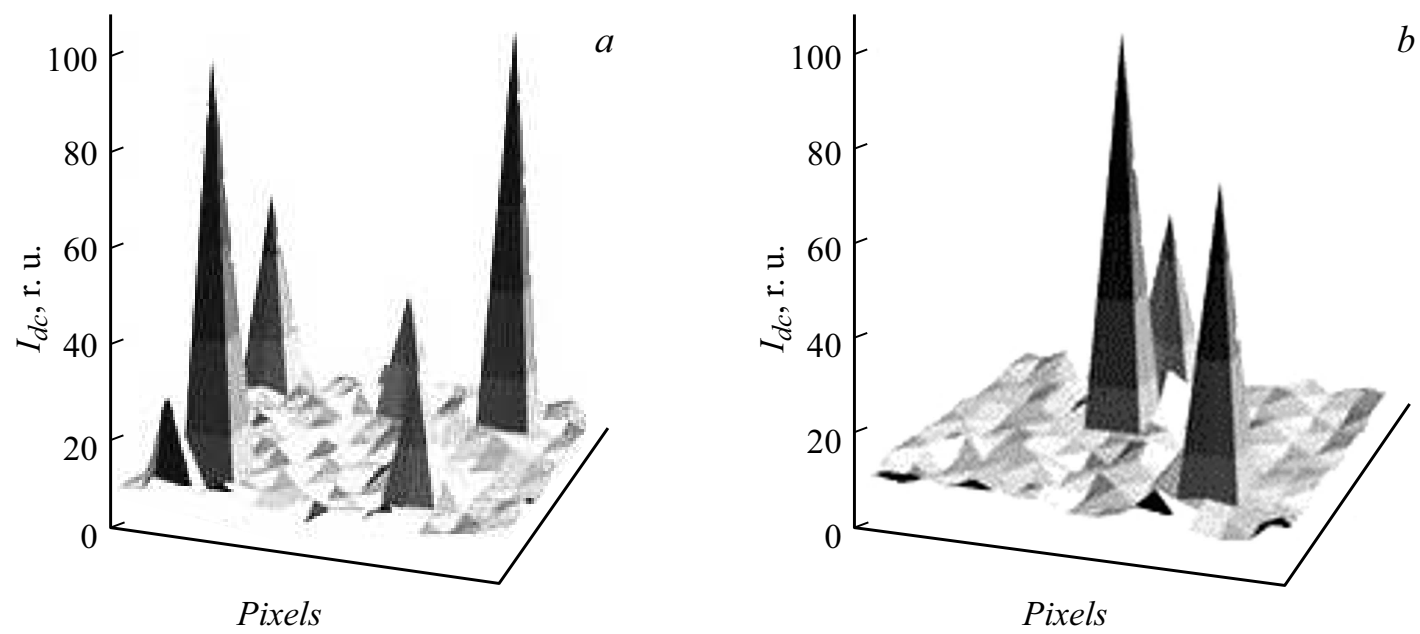

Рис. 5. Рельефы кластеров спайков в CMOS-матрице при облучении: $a$ - протонами до флюенса $10^{10} \mathrm{~cm}^{-2}, b-$ нейтронами до флюенса $2.8 \cdot 10^{7} \mathrm{~cm}^{-2}$.
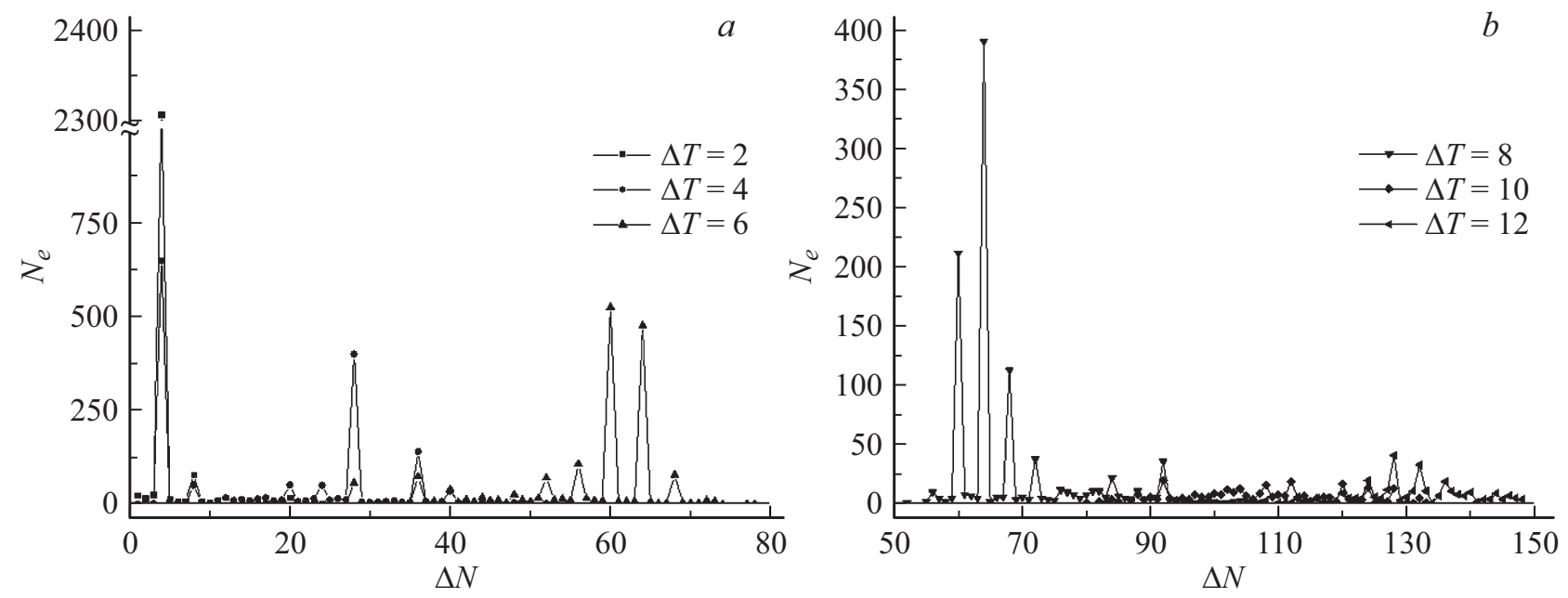

Рис. 6. Распределение числа пар последовательных сбоев $N_{e}$ в зависимости от интервалов в их адресах $\Delta N$ для временных интервалов между ними $\Delta T=2,4,6(a)$ и $\Delta T=8,10,12 \mathrm{~ms}(b)$ для образца № 1 SRAM.

В процессе облучения регистрировались время и адрес сбоя поврежденного байта. Анализ полученных данных показал, что в пределах каждого цикла чтения всего объема памяти существуют сбои с малой разницей во времени регистрации $(\Delta T)$, а также малой и характерно кратной разницей в адресе $(\Delta N)$ байтов. На рис. 6 приведены распределения количества последовательных сбоев $N_{e}$ в образце SRAM, идущих через определенные временные интервалы $\Delta T$, в зависимости от разницы в адресах поврежденных ячеек $\Delta N$. Кратность временных интервалов величине $2 \mathrm{~ms}$ между сбоями обусловлена минимальным периодом обработки информации опрашивающего устройства. Выбор конкретных значений $\Delta T$ объясняется наибольшей статистикой сбоев в диапазоне $\Delta T$ от 2 до $12 \mathrm{~ms}$.

Из рис. 6 видно, что имеют место ярко выраженные максимумы при $\Delta N=4,28,60,64$, означающие, что близкие по времени образования сбои разнесены друг
Таблица 3. Количество „кластеров“ $N$ в зависимости от количества входящих в состав кластера сбоев $n$ для образца SRAM № 1 при условии $\Delta T \leq 8 \mathrm{~ms}$ и $\Delta N \leq 92$ byte (на $10^{4}$ сбоев)

\begin{tabular}{c|c|c|c|c|c|c|c|c|c|c}
\hline$n$ & 2 & 3 & 4 & 5 & 6 & 7 & 8 & 9 & 11 & 12 \\
\hline$N$ & 695 & 331 & 124 & 62 & 42 & 20 & 9 & 8 & 4 & 4
\end{tabular}

относительно друга преимущественно на определенные величины $\Delta N$. Наличие таких максимумов указывает на возможность возникновения групп сбоев в байтах, расположенных близко друг от друга. По экспериментальным данным определены распределения количества таких групп по числу одиночных сбоев, входящих в их состав. В состав групп были включены сбои со значениями интервалов $\Delta T \leq 8,10$ и $12 \mathrm{~ms}$ и с разницей в адресах $\Delta N \leq 92,128$ и 148 соответственно. В табл. 3 


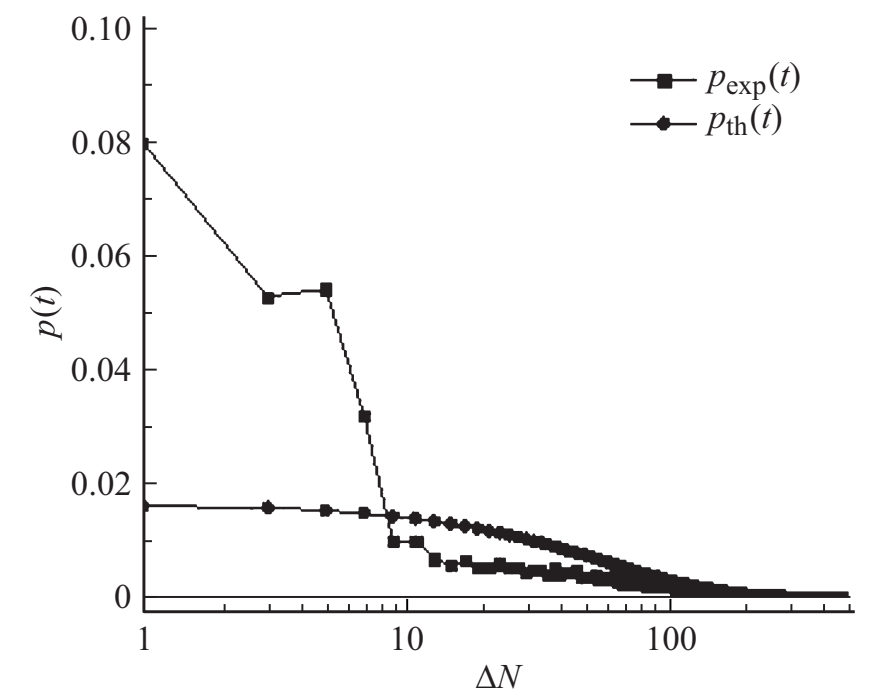

Рис. 7. Теоретическая $p_{\mathrm{th}}(t)$ и экспериментальная $p_{\exp }(t)$ зависимости вероятностей интервалов времени между сбоями для образца SRAM № 1.

приведены такие данные в образце SRAM № 1 при условии $\Delta T \leq 8 \mathrm{~ms}$ и $\Delta N \leq 92$ byte. Из таблицы видно, что количество последовательных сбоев при выбранных граничных условиях в группах может составлять свыше 10, а доля сбоев, входящих в состав групп, достигает $35 \%$. К образованию групп таких размеров, так же как и в матрицах, способны приводить остаточные ядра из ядерных реакций нуклонов с ядрами кремния (табл. 1).

Рассмотренные группы сбоев могут представлять собой МС. В связи с отсутствием информации о топологии исследуемых SRAM для подтверждения возможности образования MC был использован статистический анализ временных промежутков между сбоями. При отсутствии эффекта образования МС временной интервал между созданием отдельных сбоев является случайным событием и теоретическая вероятность того, что интервал времени между двумя последовательными сбоями лежит в пределах от $t$ до $t^{+} \Delta t$ равна [24]

$$
p_{\text {th }}(t)=n_{0} \exp \left(-n_{0} t\right) \Delta t
$$

где $n_{0}=n / T_{\mathrm{ir}}$ - число сбоев в единицу времени, $T_{\mathrm{ir}}-$ продолжительность облучения, $n_{0} \exp \left(-n_{0} t\right)$ - плотность распределения вероятностей интервалов $t$ между сбоями.

Для образца SRAM № 1 величина $n_{0}$ равна $n_{0}=n / T_{\text {ir }}$ $=16169 / 767.4-1=0.0164 \mathrm{~s}^{-1}$. Соответственно теоретическая зависимость плотности распределения вероятности случайных сбоев имеет вид: $p_{\text {th }}(t)=$ $=0.0164 n_{0} \exp (-0.0164 t)$. Экспериментальные данные по распределению времени между сбоями $\Delta T$ для образца SRAM № 1 приведены в табл. 4. На рис. 7 представлены теоретическая $p_{\mathrm{th}}(t)(1)$ и полученная по данным табл. 4 экспериментальная $p_{\exp }(t)$ зависимости плотности вероятности от времени облучения. Превышение
Таблица 4. Распределение сбоев по времени между сбоями $\Delta T$ для образца SRAM № 1

\begin{tabular}{c|c|c|c|c|c|c|c|c|c|c|c}
\hline$\Delta T, \mathrm{~ms}$ & 2 & 4 & 6 & 8 & 10 & 14 & 18 & 22 & 24 & 28 & 32 \\
\hline$N$ & 2590 & 1712 & 1755 & 1037 & 321 & 223 & 214 & 171 & 195 & 173 & 160 \\
\hline$\Delta T, \mathrm{mc}$ & 34 & 36 & 38 & 40 & 42 & 46 & 50 & 54 & 56 & 60 & 64 \\
\hline$N$ & 161 & 134 & 169 & 142 & 144 & 156 & 118 & 127 & 108 & 124 & 126
\end{tabular}

экспериментальной зависимости $p_{\exp }(t)$ над теоретической $p_{\text {th }}(t)$ означает то, что значительная часть сбоев, по крайней мере при $\Delta T \leq 10 \mathrm{~ms}$, не является случайными независимыми событиями и входит в состав МС. По нашим оценкам, при $\Delta T \leq 10 \mathrm{~ms}$ около $50 \%$ всех сбоев содержатся в МС, что согласуется с данными работ $[9,25]$.

\section{Заключение}

Проведенные исследования показали, что:

- под действием быстрых протонов и нейтронов в светочувствительных ИС образуются кластеры, включающие до 14 соседних спайков, которые представляют собой аналог МС в ИС памяти;

- под действием быстрых протонов в изделиях памяти SRAM возникают MC, включающие до 10 байтов (в заданных граничных условиях);

- механизм образования кластеров в матрицах и МС изделиях памяти под действием быстрых нуклонов аналогичен и обусловлен вторичными частицами, возникающими в результате ядерных реакций нуклонов с ядрами атомов кремния;

- предложенный статистический анализ временных промежутков между сбоями может быть полезным методическим подходом при анализе экспериментальных данных на предмет выявления МС при отсутствии данных о топологии ИС;

- в облученных матрицах размеры кластеров и величины темновых токов спайков, входящих в их состав, практически не зависят от типа нуклонов;

- в исследованных матрицах и изделиях памяти SRAM значительная часть повреждений (до 50\%) входит в состав кластеров и МС.

Возможность образования в изделиях памяти SRAM множественных сбоев должна учитываться при разработке электронной аппаратуры, а именно

- необходимо разрабатывать методы парирования таких эффектов,

- становится недопустимым использовать ячейки одной и той же ИС на разных параллельных информационных каналах.

\section{Конфликт интересов}

Авторы заявляют, что у них нет конфликта интересов. 


\section{Список литературы}

[1] Srour J.R., Hartmann R.A., Kitazaki K.S. // IEEE Trans. Nucl. Sci. 1986. Vol. 33. Dec. P. 1597-1604.

[2] Srour J., Marshall C.J., Marshall P.W. // IEEE Trans. Nucl. Sci. 2003. Vol. 50. Jan. P. 653-670.

[3] Чумаков А.И. Действие космической радиации на интегральные схемы. М.: Радио и связь, 2004. 320 с.

[4] Janni J.F. // Atomic data and nuclear data tables. 1982. Vol. 27. P. 147-529.

[5] Messenger R., Burke E.A., Xapsos M.A., Summers G.P., Walters R.J., Jun I., Jordan T. // IEEE Trans. Nucl. Sci. 2003. Vol. 50. Dec. P. 1929-1933.

[6] Gereth R.H., Haitz, Smits F.M. // J. Appl. Phys. 1965. Vol. 36. N 12. Dec. P. 3884-3894.

[7] Marshall P., Marshall C. // Proc. Short Course NSREC. 1999. P. 51-100.

[8] Pickel A.H., Calmfa G.R., Hopkinson C.J. Marshall // IEEE Trans. Nucl. Sci. 2003. Vol. 50. June. P. 671-688.

[9] Mavis D.G., Eaton P.H., Sibley M.D., Lacoe R.C., Smith E.J., Avery K.A. // IEEE Trans. Nucl. Sci. 2008. Vol. 55. N 6. Dec. P. 3288-3294.

[10] Hopkinson G., Dale C.J., Marshall P.W. // IEEE Trans. Nucl. Sci. 1996. Vol. 43. Apr. P. 614-627.

[11] Абросимов Н.К., Воробьев А.С., Иванов Е.М., Михеев Г.Ф., Рябов Г.А., Тверской М.Г, Щербаков О.А. // ВАНТ. Сер. Физика радиационного воздействия на радиоэлектронную аппаратуру. Научно-технический сборник. 2010. Вып. 4. C. $107-112$.

[12] Иванов Н.А., Лобанов О.В., Пашук В.В. // ПТЭ. 2009. № 6. C. 5-10. [Ivanov N.A., Lobanov O.V., Pashuk V.V. // Instruments and Experiment. Techniq. 2009. Vol. 52. N 6. P. 763-768.]

[13] Cumming J.B. // Phys. Rev. 1963. Vol. 13. P. 261.

[14] Measurement and reporting of alpha particle and terrestrial cosmic ray-induced soft errors in semiconductor devices. JEDEC Standard - JESD89A, Oct. 2006.

[15] Абросимов Н.К., Вайшнене Л.А., Воробьёв А.С., Иванов Е.М., Михеев Г.Ф., Рябов Г.А., Тверской М.Г., Щербаков O.A. // ПТЭ. 2010. № 4. C. 5-12. [Abrosimov N.K., Vaishnene L.A., Vorob'ev A.S., Ivanov E.M., Mikheev G.F., Ryabov G.A., Tverskoi M.G., Shcherbakov O.A. // Instrum. Experiment. Techniq. 2010. Vol. 53. N 4. P. 469-476.]

[16] Ермаков К.Н., Иванов Н.А., Лобанов О.В., Пашук В.В., Тверской М.Г., Любинский С.М. // Письма в ЖТФ. 2010. T. 36. Вып. 13. С. 54-60. [Ermakov K.N., Ivanov N.A., Lobanov O.V., Pashuk V.V., Tverskoy M.G., Lyubinskii S.M. // Tech. Phys. Lett. 2010. Vol. 36. N 7. P. 610-612.]

[17] Иванов Н.А., Лобанов О.В., Митин Е.В., Пашук В.В., Тверской М.Г. // Письма в ЖТФ. 2013. Т. 39. Вып. 17. C. 35-43. [Ivanov N.A., Lobanov O.V., Mitin E.V., Pashuk V.V., Tverskoy M.G. // Techn. Phys. Lett. 2013. Vol. 39. N 9. P. 711774].

[18] Иванов Н.А., Пашук В.В., Понежка Т.Е., Тверской М.Г. // ВАНТ. Сер. „Физика радиационного воздействия на радиоэлектронную аппаратуру“, Научно-технический сборник. 2012. Вып. 2 (апрель-июнь). С. 61-63.

[19] Ермаков К.Н., Иванов Н.А., Маркелов В.В., Пашук В.В., Тверской М.Г. // ВАНТ. Сер. „Физика радиационного воздействия на радиоэлектронную аппаратуру“. Научнотехнический сборник. 2006. Вып. 1-2. С. 54-57.
[20] Sirianni M., Mutchler M., Clampin M., Ford $H$., Illingworth G., Hartig G., Van Orsow D., Wheeler T. // Proc. SPIE. 2004. Vol. 5499. P. 173-184.

[21] Dawson K., Bebek C., Emes J., Holland S., Jelinsky S., Karcher A., Kolbe W., Palaio N., Roe N., Saha J., Takasaki K., Wang G. // IEEE Trans. Nucl. Sci. 2008. Vol. 55. N 3. P. 17251735.

[22] Барашенков В.С. Сечения взаимодействия частиц и ядер с ядрами. Дубна: ОИЯИ, 1993. 346 с.

[23] Cannon E.H., Cabanas-Holmen M., Wert J., Amort T., Brees R., Koehn J., Meaker B., Normand E. // IEEE Trans. Nucl. Sci. 2010. Vol. 57. N 6. Dec. P. 3493-3499.

[24] Калашников В.И., Козодаев М.С. Детекторы элементарных частиц. М.: Наука, 1966. С. 246.

[25] Secondo R., Foucard G., Danzeca S., Losito R., Peronnard P., Masi A., Brugger M., Dusseau L. RADECS 2015. Moscow. Russia. 14-18 September. 